\title{
The Relationship Between Global Sleep Score And Inflammatory Markers In Obese Adults From The United States
}

This article was published in the following Dove Press journal:

Nature and Science of Sleep

\author{
Ying Huang ${ }^{l} *$ \\ Ying Jiang ${ }^{2, *}$ \\ Meilan Zhu ${ }^{2}$ \\ 'Department of Cardiovascular Medicine, \\ The Second Affiliated Hospital of \\ Nanchang University, Nanchang, Jiangxi \\ 330006, People's Republic of China; \\ ${ }^{2}$ Department of Rehabilitation, The \\ Second Affiliated Hospital of Nanchang \\ University, Nanchang, Jiangxi 330006, \\ People's Republic of China
}

*These authors contributed equally to this work
Correspondence: Meilan Zhu Department of Rehabilitation, The Second Affiliated Hospital of Nanchang University, No. I Minde Road, Donghu

District, Nanchang, Jiangxi 330006,

People's Republic of China

Email15070896289@I39.com
Background: Poor sleep is a risk factor for cardiovascular diseases (CVDs). The underlying pathogenesis is not clear. Levels of inflammatory markers, such as C-reactive protein (CRP), interleukin-6 (IL-6) and tumor necrosis factor-a (TNF-a), have been found to be elevated in patients with CVDs.

Aim: The study aimed to investigate the associations between sleep quality and serum inflammatory markers in a cohort of obese adults.

Methods: This was a second analysis of the data from the Midlife in the United States (MIDUS) study, a longitudinal study of a national (US) sample of adults. A total of 1255 participants completed comprehensive biological assessments. The associations between global sleep score and serum levels of inflammatory markers were analyzed.

Results: Univariate analysis showed that a higher global sleep score was correlated with lower age $(r=-0.079, \mathrm{P}=0.009)$, higher BMI $(r=0.100, \mathrm{P}=0.001)$ and heavier perceived stress $(r=0.335$, $\mathrm{P}<0.001)$. Multivariate linear regression analysis showed that the global sleep score was positively related to levels of IL-6 ( $\mathrm{S} \beta=0.074, \mathrm{P}=0.009), \mathrm{IL}-8(\mathrm{~S} \beta=0.089, \mathrm{P}=0.002), \mathrm{TNF}-\mathrm{a}(\mathrm{S} \beta=0.0 .082$, $\mathrm{P}=0.005)$, E-selectin $(\mathrm{S} \beta=0.071, \mathrm{P}=0.016)$ and intercellular adhesion molecule-1 (ICAM-1, $\mathrm{S} \beta=0.117, \mathrm{P}<0.001$ ) after adjustments were made for age, gender, race, marital status, education, current smoking status, physician-diagnosed CVDs and respiratory diseases, BMI and perceived stress. However, the global sleep score was not associated with serum IL-10 ( $\mathrm{S} \beta=-0.021, \mathrm{P}=0.463)$ and $\mathrm{CRP}(\mathrm{S} \beta=0.035, \mathrm{P}=0.059)$ levels after adjustments were made for these confounding factors. Conclusion: Poor sleep is positively associated with serum inflammatory marker levels among obese adults. Sufficient sleep may be particularly important for obese adults to prevent CVDs.

Keywords: global sleep score, inflammation, body mass index, perceived stress, obese adults

\section{Introduction}

Concern for sleep problems as risk factors for adverse health has grown since an association between poor sleep and mortality was first described over four decades ago. ${ }^{1}$ Studies have generally shown a significantly positive relationship between poor sleep and mortality. In other words, people with poor sleep have a greater mortality risk than those with higher sleep quality. Many publications have begun reporting that poor sleep can predict a growing number of diseases. ${ }^{2,3}$ In recent years, however, due to increasingly social and economic factors, shorter sleep time, poor sleep quality and other sleep problems have become increasingly serious. ${ }^{4-6}$ 
Poor sleep is related to poor decision making, risk taking and mood disruptions. ${ }^{7-10}$ The direct effect of poor sleep is tiredness during low-stimulation activities, including attentiveness while studying, driving, performing repetitive activities and engaging in other activities. ${ }^{7}$ Many investigations have shown that sleep in the classroom has become a very common phenomenon in many high schools. ${ }^{7}$ A previous study showed that poor-quality sleep and insufficient sleep are pervasive in the general population and are linked with impairments in cognitive control and greater risk taking. ${ }^{8}$ Moreover, some other studies suggested that another important effect of sleep deprivation is related to mood. Relationships have been found between poor sleep and depression or mood lability. ${ }^{9,10}$

Although the negative effects of sleep problems on psychosocial and behavioral problems are well understood, less is known about the relationship between sleep and biomarkers of physical health in the general population. It is well known that inflammation has attracted increasing attention and is considered a biomarker of risk for several chronic adult health conditions, such as cardiovascular diseases (CVDs), that may be associated with sleep problems. ${ }^{11-15}$ Research has suggested that chronic sleep problems lead to increased inflammation levels, such as C-reactive protein (CRP), among adults. ${ }^{16-18}$ Some studies have also shown that disrupted sleep has negative effects on the production and activation of cells involved in immune regulation, causing an alteration of inflammatory factors. ${ }^{19,20}$ Therefore, the aim of this study was to assess the relationships between global sleep score and systemic inflammatory factors in a comprehensive and multi-level manner.

\section{Materials And Methods Study Population}

This was a reanalysis of the data from the Midlife in the United States (MIDUS) study, a longitudinal study of a national (US) sample of adults aged 34-84 years at baseline. The MIDUS study investigates the association between psychological, behavioral, and social factors and age-related physical and mental health problems. As a sub-component of the MIDUS study, 1255 participants completed the Biomarkers Project, in which participants provided comprehensive biological assessments as a way to integrate behavioral and psychosocial factors with biology. ${ }^{21}$ The measures described below were included to best capture our contents of interest.

Full details of the MIDUS study biomarker protocol are available elsewhere. ${ }^{21,22}$ Complete data and specific codebooks are also available at http://www.midus.wisc. edu/. In summary, participants in the MIDUS study were originally recruited in 1995-1996 by means of a national sample collected by random-digit dialing procedures. To be as inclusive as possible, all living participants in the first MIDUS survey who could safely go to the clinic were considered eligible for participation in the Biomarkers Project. They were recruited to participate using e-mail and follow-up phone calls. Data were collected between 2004 and 2009 at one of three affiliated General Clinical Research Centers of the MIDUS study (University of Wisconsin-Madison; Georgetown University; University of California-Los Angeles). By using a standardized protocol that was consistent across the three sites, participants completed detailed self-administered questionnaires, medical history interviews, and the collection of blood specimens during a 2-day visit. Each participant was remunerated \$200 for participating, and traveling expenses were covered. The Biomarkers Project protocol was approved by the institutional review boards of each General Clinical Research Center, and all participants gave written informed consent.

Serum samples from all participants were collected and tested during a 2-day visit between 2004 and 2009. The following serum inflammatory markers were measured: CRP, interleukin-6 (IL-6), IL-8, IL-10, E-selectin, intracellular adhesion molecule-1 (ICAM-1), and tumor necrosis factor-a (TNF-a). These serum biomarkers were collected during a medical exam at one of three General Clinical Research Centers. Biomarkers were obtained from a fasting blood draw. Full measurement methods have been described in detail elsewhere. ${ }^{21,22}$ In this study, body mass index $(\mathrm{BMI}) \geq 25$ was defined as overweight or obesity. For our purposes, all included participants were overweight or obese adults.

\section{Global Sleep Score}

The global sleep score was measured with the Pittsburgh Sleep Quality Index (PSQI). ${ }^{23}$ The PSQI is widely used and a reliable measure of global sleep quality and sleep disturbances over the past month. The 19 items are divided into seven component scores that reflect the frequency of sleep problems in the following areas: subjective sleep quality, sleep latency, sleep duration, 
habitual sleep efficiency, sleep disturbance, use of sleep medication, and daytime dysfunction. A global sleep score ranging from 0 to 21 can be obtained by summing the seven components after weighting them on a scale ranging from 0 to $3[a=0.74]$. For each component as well as the global score, higher scores show worse sleep quality. ${ }^{23}$

\section{Perceived Psychological Stress}

The Perceived Stress Scale (PSS) is a 10-item measure that can assess the degree of pressure on participants in their lives. ${ }^{24}$ Each item (e.g., "In the past month, how often have you been upset because of something that happened unexpectedly?") used a 5-point scale ranging from 1 (never) to 5 (very often), and items were reversecoded as needed so that higher scores show greater perceived stress $[\mathrm{a}=0.84]$.

\section{Statistical Analysis}

The normality of the data was analyzed by the Kolmogorov-Smirnov test combined with Q-Q plots. The data that were not normally distributed were expressed as the median (interquartile range [IQR]), and data with normal distributions were expressed as the mean \pm standard deviation $(\mathrm{M} \pm \mathrm{SD})$. First, the correlations between global sleep score and age, BMI and perceived stress were computed with Spearman correlation analysis in this study. Then, all variables with non-normal distributions were standardized with z-scores and analyzed with Pearson's correlation coefficient. The associations between global sleep score and serum inflammatory markers were further assessed by multivariate linear regression analysis. All of the analyses were performed using R3.4.4 and SPSS. $P \leq 0.05$ was considered to be statistically significant.

\section{Results}

\section{The Characteristics Of The Study Subjects}

Due to the lack of complete serum test data for 148 participants during the follow-up period, data for the remaining 1107 participants were included in the study and further analyzed.

Table 1 presents the $\mathrm{N}(\%), \mathrm{M} \pm \mathrm{SD}$ and median (IQR) for the variables. The sociodemographic characteristics were as follows: 474 participants $(42.8 \%)$ were males, 1018 participants $(92.0 \%)$ were white, 702 participants $(63.4 \%)$ were married, 443 participants $(40.0 \%)$ had
Table I Participant Characteristics

\begin{tabular}{|c|c|}
\hline Variables & $\begin{array}{l}\mathbf{N}(\%) \text { or } M(I Q R) \text { or } M \\
\pm S D\end{array}$ \\
\hline \multicolumn{2}{|l|}{ Sociodemographic characteristics } \\
\hline Age (years) & $54(45-63)$ \\
\hline Gender (\% male) & $474(42.8 \%)$ \\
\hline Race (\% white) & $1018(92.0 \%)$ \\
\hline Marital status (married), n (\%) & $702(63.4 \%)$ \\
\hline $\begin{array}{l}\text { Education (with bachelor's degree or } \\
\text { higher), } n(\%)\end{array}$ & $443(40.0 \%)$ \\
\hline Current smoker, n (\%) & $163(14.6 \%)$ \\
\hline BMI & $28.59(26.13-33.05)$ \\
\hline Perceived stress scale & $22(18-26)$ \\
\hline Global Sleep Score & $6.21 \pm 3.67$ \\
\hline Subjective Sleep Quality & $1.00 \pm 0.69$ \\
\hline Sleep Latency & $0.95 \pm 0.94$ \\
\hline Sleep Duration & $0.84 \pm 0.80$ \\
\hline Habitual Sleep Efficiency & $0.69 \pm 1.06$ \\
\hline Sleep Disturbances Range & $1.32 \pm 0.58$ \\
\hline Sleeping Medication & $0.58 \pm 1.08$ \\
\hline Daytime Dysfunction & $0.83 \pm 0.67$ \\
\hline \multicolumn{2}{|l|}{$\begin{array}{l}\text { Physician diagnosed cardiovascular } \\
\text { diseases }\end{array}$} \\
\hline Heart diseasen, (\%) & $126(11.4 \%)$ \\
\hline TIA or stroke, (\%) & $43(3.9 \%)$ \\
\hline Diabetes, (\%) & 132 (|I.9\%) \\
\hline High blood pressure, (\%) & $402(36.3 \%)$ \\
\hline \multicolumn{2}{|l|}{ Physician diagnosed respiratory diseases } \\
\hline Asthma, n (\%) & $127(\mid 1.7 \%)$ \\
\hline Emphysema/COPD, n (\%) & $29(2.6 \%)$ \\
\hline \multicolumn{2}{|l|}{ Serum inflammatory biomarkers } \\
\hline IL-6 (pg/mL) & $0.80(0.56-1.22)$ \\
\hline IL-8 (pg/mL) & $12.40(9.13-15.79)$ \\
\hline IL-I0 (pg/mL) & $0.22(0.17-0.32)$ \\
\hline CRP (ug/mL) & I.43 (0.69-3.66) \\
\hline TNF-a (pg/mL) & $2.05(1.69-2.5 I)$ \\
\hline E-Selectin (ng/mL) & 39.11 (28.09-5I.85) \\
\hline ICAM-I (ng/mL) & $273.92(221.03-336.05)$ \\
\hline
\end{tabular}

Notes: $M($ (IQR) for nonnormally distributed data, $M \pm S D$ for normally distributed data and $\mathrm{n}(\%)$ for categoric variables.

Abbreviations: BMI, body mass index; COPD, chronic obstructive pulmonary disease; IL, Interleukin; CRP, C-reactive protein, TNF-a, tumor necrosis factor-a; ICAM-I, intercellular adhesion molecule-I; M (IQR), median interquartile range; $M \pm S D$, mean \pm standard deviation.

bachelor's degrees or higher, and 163 participants $(14.6 \%)$ were current smokers. The median age of the entire population in this study was 54 years. A higher global sleep score was correlated with younger age $(r=-0.079, \mathrm{P}=0.009$, Figure 1$)$, higher BMI $(r=0.100$, $\mathrm{P}=0.001$, Figure 2) and heavier perceived stress $(r=0.335, \mathrm{P}<0.001$, Figure 3$)$. 


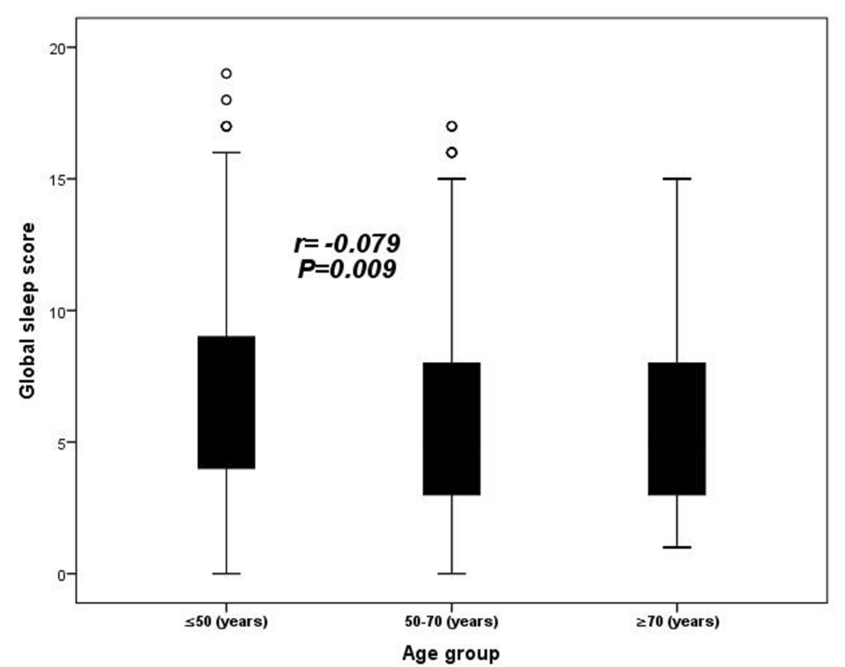

Figure I The relationship between global sleep score and age in all subjects.

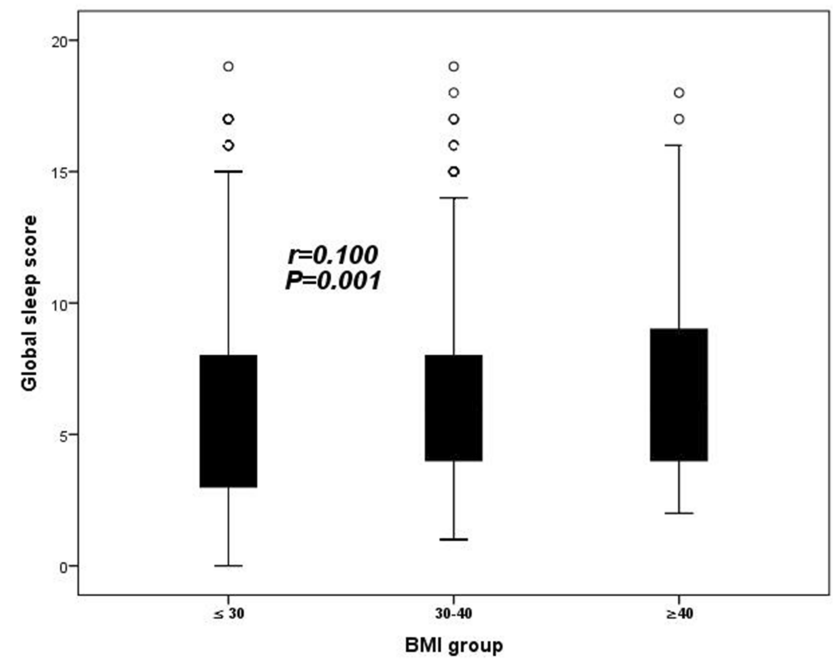

Figure 2 The relationship between global sleep score and BMI in all subjects.

\section{Relationships Between Sleep And Inflammation Markers By Univariate}

\section{Analysis}

As shown in Table 2, higher global sleep scores were associated with higher serum levels of all inflammation markers in this study except IL-10 (all $\mathrm{P}<0.05$ ). Worse subjective sleep quality was related to higher levels of TNF-a, E-selectin and ICAM-1 (all $\mathrm{P}<0.05$ ). Longer sleep latency showed a positive association with IL-6, CRP and ICAM-1 levels (all $\mathrm{P}<0.05$ ), and shorter sleep duration was associated with higher levels of CRP, E-selectin and ICAM-1 (all $\mathrm{P}<0.05$ ). Worse habitual sleep efficiency was positively related to levels of IL-6, IL-8, CRP, E-selectin and ICAM-1 (all P<0.05). Those

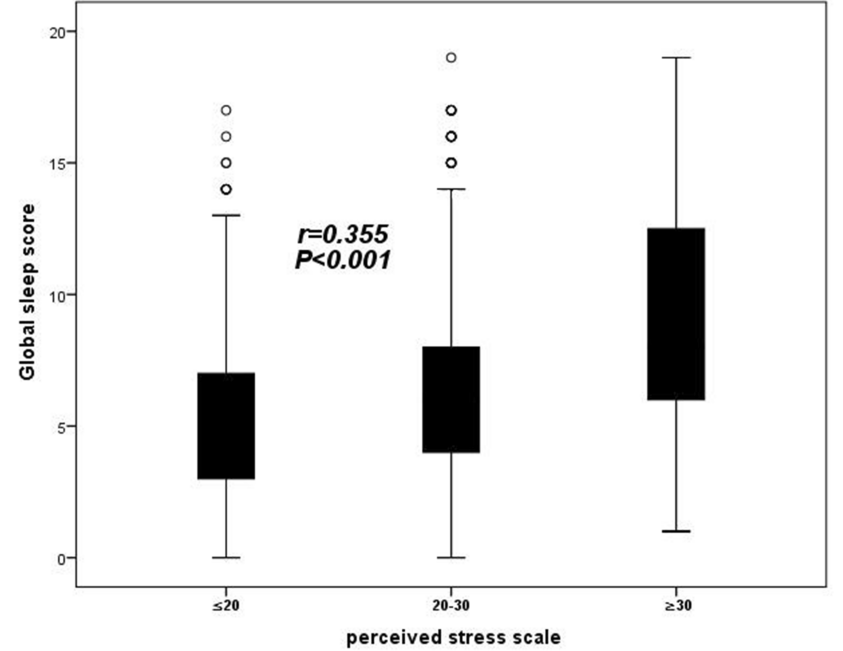

Figure 3 The relationship between global sleep score and perceived stress in all subjects.

with a greater range of sleep disturbances had higher IL8, TNF- $\mathrm{a}$, CRP and ICAM-1 levels (all $\mathrm{P}<0.05$ ). The frequency of sleeping medication use was positively associated with IL-6, IL8 and ICAM-1 (all P<0.05), and the frequency of daytime dysfunction did not show a positive relationship with inflammation markers (all $\mathrm{P}>0.05$ ). These results showed that worse sleep quality was associated with higher serum inflammation levels by Pearson correlation analysis. However, the 7 factors were not associated with serum IL-10 levels.

\section{Associations Between Global Sleep Score and Inflammatory Markers After Adjusting For Confounding Factors}

To further prove the correlation between sleep quality and serum inflammatory biomarkers, multiple linear regression analysis was performed. As shown in Table 3, the global sleep score was positively related to IL- $6(S \beta=0.095$, $\mathrm{P}=0.001)$, IL-8 $(\mathrm{S} \beta=0.097, \mathrm{P}=0.001), \mathrm{CRP} \quad(\mathrm{S} \beta=0.106$, $\mathrm{P}<0.001), \quad$ TNF-a $\quad(\mathrm{S} \beta=0.102, \quad \mathrm{P}=0.001), \quad$ E-selectin $(\mathrm{S} \beta=0.114, \mathrm{P}<0.001)$ and ICAM-1 $(\mathrm{S} \beta=0.140, \mathrm{P}<0.001)$ after adjusting for age, gender, race, marital status, education, current smoking status and physician-diagnosed CVDs and respiratory diseases (Model 1). After other confounding factors were added, including BMI and perceived stress in addition to CRP, the global sleep score still had a strong relationship with these inflammatory biomarkers (all $\mathrm{P}<0.05$, Model 3). However, consistent with univariate analysis, the global sleep score was not associated with serum levels of IL-10 after adjustments were made 
Table 2 Bivariate Correlations Using Standardized Variables

\begin{tabular}{|c|c|c|c|c|c|c|c|c|c|c|c|c|c|c|}
\hline Variables & $\mathbf{I}$ & 2 & 3 & 4 & 5 & 6 & 7 & 8 & 9 & 10 & II & 12 & 13 & 14 \\
\hline \multicolumn{15}{|l|}{ I. Global Sleep Score } \\
\hline 2. Subjective Sleep Quality & $0.70^{\#}$ & & & & & & & & & & & & & \\
\hline 3. Sleep Latency & $0.70^{\#}$ & $0.42^{\#}$ & & & & & & & & & & & & \\
\hline 4. Sleep Duration & $0.64^{\#}$ & $0.42^{\#}$ & $0.28^{\#}$ & & & & & & & & & & & \\
\hline 5. Habitual Sleep Efficiency & $0.7 I^{\#}$ & $0.4 I^{\#}$ & $0.39^{\#}$ & $0.53^{\#}$ & & & & & & & & & & \\
\hline 6. Sleep Disturbances & $0.55^{\#}$ & $0.39^{\#}$ & $0.36^{\#}$ & $0.21^{\#}$ & $0.22^{\#}$ & & & & & & & & & \\
\hline \multicolumn{15}{|l|}{ Range } \\
\hline 7. Sleeping Medication & $0.56^{\#}$ & $0.23^{\#}$ & $0.31^{\#}$ & $0.12^{\#}$ & $0.19^{\#}$ & $0.21^{\#}$ & & & & & & & & \\
\hline 8. Daytime Dysfunction & $0.53^{\#}$ & $0.38^{\#}$ & $0.24^{\#}$ & $0.27^{\#}$ & $0.21^{\#}$ & $0.32^{\#}$ & $0.17^{\#}$ & & & & & & & \\
\hline 9. IL-6 & $0.10^{8}$ & 0.06 & $0.08^{8}$ & 0.02 & $0.09^{\&}$ & 0.04 & $0.08^{\&}$ & 0.04 & & & & & & \\
\hline 10. IL-8 & $0.09^{\&}$ & 0.04 & 0.06 & 0.05 & $0.07^{*}$ & $0.07^{*}$ & $0.07^{*}$ & 0.05 & 0.06 & & & & & \\
\hline II. IL-10 & -0.02 & -0.06 & 0.00 & -0.02 & -0.02 & -0.01 & -0.01 & 0.01 & 0.03 & 0.02 & & & & \\
\hline 12. TNF-a & $0.07^{*}$ & $0.06 *$ & 0.04 & 0.04 & 0.04 & $0.06 *$ & 0.05 & 0.04 & 0.05 & $0.18^{\#}$ & $0.08^{\&}$ & & & \\
\hline 13. CRP & $0.12^{\#}$ & 0.05 & $0.09^{\&}$ & $0.08^{8}$ & $0.12^{\#}$ & $0.06 *$ & 0.05 & 0.06 & $0.08^{\&}$ & 0.02 & $0.20^{\#}$ & $0.17^{\#}$ & & \\
\hline 14. E-Selectin & $0.11^{\#}$ & $0.08^{8}$ & 0.05 & $0.11^{\#}$ & $0.09^{\&}$ & 0.03 & 0.04 & 0.09 & 0.08 & $0.12^{\#}$ & 0.03 & $0.08^{8}$ & $0.15^{8}$ & \\
\hline I5. ICAM-I & $0.13^{\#}$ & $0.09^{2}$ & $0.07 *$ & $0.07^{\&}$ & $0.10^{\#}$ & $0.10^{\#}$ & $0.08^{\&}$ & 0.05 & 0.02 & $0.07 *$ & $0.08^{\&}$ & $0.29^{8}$ & $0.15^{\&}$ & $0.07^{*}$ \\
\hline
\end{tabular}

Notes: ${ }^{*} P<0.05,{ }^{\&} P<0.01,{ }^{\#} P<0.001$. Pearson correlation analysis was used.

Abbreviations: IL, Interleukin; CRP, C-reactive protein, TNF-a, tumor necrosis factor-a; ICAM-I, intercellular adhesion molecule-I.

for these confounding factors in multivariate analysis (all $\mathrm{P}>0.05$, Model 3).

\section{Discussion}

This study with obese adults aimed to investigate the associations between sleep quality and key biomarkers of inflammation that predict chronic health problems, such as CVDs, in adulthood. In addition to daytime dysfunction, the other 6 indicators from the PSQI were strongly associated with inflammatory factors by univariate analysis. Higher global sleep scores were significantly linked to higher levels of inflammatory factors after adjustments were made for other confounding factors in multivariate analysis.

Sleep-related research has been ongoing for more than 40 years. Poor sleep is a risk factor for CVDs. ${ }^{25-30}$ A large number of studies have shown that poor sleep and sleeprelated diseases play an important role in the morbidity and mortality of adults, but these studies cannot prove this association. ${ }^{31-33}$ Few studies have tried to establish a model of long-term partial sleep deprivation that can best reflect the daily decline in sleep quality. These studies did not include a comprehensive sleep score, had insufficient inflammatory indicators, had insufficient correction for confounding factors, had flawed research designs and had unrepresentative research populations..$^{11-15,34,35}$ Thus, to achieve this goal, well-designed prospective studies with assessments of all aspects of sleep, inflammation, many confounding factors of sleep and associated risks are necessary. Our research has met these conditions. The results of our study contribute to the literature in three different ways. On the one hand, the data of this study were from the MIDUS study, a longitudinal investigation of a national (US) sample of adults. Multiple aspects of sleep quality were assessed, thus expanding upon existing research on the association between adult sleep and inflammation. ${ }^{13-15}$ On the other hand, our results via multivariate analysis first proved that sleep quality, as evaluated with the global sleep score, was significantly and independently associated with levels of inflammatory factors, including IL-6, IL-8, TNF-a, E-selectin and ICAM-1. These serum inflammatory markers have been proven to play an important role in the occurrence and development of CVDs among middle-aged and elderly adults. ${ }^{36-39}$ Additionally, this study was the first to explore the relationships between sleep quality and inflammatory factors in an obese population with a high risk of many diseases, such as type 2 diabetes mellitus and CVDs. ${ }^{38,40}$ Interestingly, none of the PSQI sleep variables were associated with IL-10 in the univariate and multivariate analyses in this study. A possible explanation is that IL-10 is an anti-inflammatory factor, and the decline in sleep quality is more likely to be related to a pro-inflammatory effect instead of anti-inflammation. ${ }^{41}$ Our results are consistent with previous experimental evidence that poor sleep leads immune cells to produce higher levels of pro-inflammatory 
Table 3 Multivariate Linear Regression Analysis Of Association Between Global Sleep Scores And Inflammatory Markers

\begin{tabular}{|l|l|l|l|l|}
\hline Variables & $\mathbf{R}^{2}$ & $\mathbf{S} \boldsymbol{\beta}$ & $\mathbf{9 5 \%} \mathbf{C I}$ & $\boldsymbol{P}$ Value \\
\hline Model I & & & & \\
IL-6 & 0.025 & 0.095 & $0.037-0.153$ & 0.001 \\
IL-8 & 0.026 & 0.097 & $0.039-0.156$ & 0.001 \\
IL-I0 & 0.017 & -0.019 & $-0.078-0.039$ & 0.517 \\
CRP & 0.028 & 0.106 & $0.048-0.165$ & $<0.001$ \\
TNF-a & 0.026 & 0.102 & $0.042-0.162$ & 0.001 \\
E-Selectin & 0.029 & 0.114 & $0.056-0.173$ & $<0.001$ \\
ICAM-I & 0.036 & 0.140 & $0.082-0.199$ & $<0.001$ \\
\hline Model 2 & & & & \\
IL-6 & 0.036 & 0.088 & $0.030-0.146$ & 0.008 \\
IL-8 & 0.035 & 0.103 & $0.044-0.161$ & 0.001 \\
IL-I0 & 0.025 & 0.017 & $-0.572-0.042$ & 0.573 \\
CRP & 0.031 & 0.083 & $0.021-0.145$ & 0.009 \\
TNF-a & 0.033 & 0.090 & $0.030-0.150$ & 0.003 \\
E-Selectin & 0.034 & 0.098 & $0.038-0.158$ & 0.001 \\
ICAM-I & 0.042 & 0.086 & $0.028-0.144$ & 0.004 \\
\hline Model 3 & & & & \\
IL-6 & 0.125 & 0.074 & $0.019-0.129$ & 0.009 \\
IL-8 & 0.127 & 0.089 & $0.033-0.144$ & 0.002 \\
IL-I0 & 0.120 & -0.021 & $-0.076-0.035$ & 0.463 \\
CRP & 0.121 & 0.035 & $-0.025-0.094$ & 0.059 \\
TNF-a & 0.126 & 0.082 & $0.025-0.139$ & 0.005 \\
E-Selectin & 0.124 & 0.071 & $0.014-0.128$ & 0.016 \\
ICAM-I & 0.133 & 0.117 & $0.061-0.172$ & $<0.001$ \\
\hline
\end{tabular}

Notes: Model I: adjusted for age, gender, race, marital status, education, current smoker and physician diagnosed CVD and respiratory diseases. Model 2: adjusted for age, gender, race, marital status, education, current smoker, physician diagnosed CVD and respiratory diseases and BMI. Model 3: adjusted for age, gender, race, marital status, education, current smoker, physician diagnosed cardiovascular diseases and respiratory diseases, BMI and perceived stress. Multivariate linear regression analysis was used.

Abbreviations: IL, Interleukin; CRP, C-reactive protein, TNF-a, tumor necrosis factor-a; ICAM-I, intercellular adhesion molecule-I; CVD, cardiovascular diseases; BMI, body mass index.

factors when stimulated in vitro. For instance, a study showed that sleep restriction with one night of $3.5 \mathrm{~h}$ of sleep was related to a significant increase in monocytederived production of TNF-a and IL- $1 \beta .{ }^{42}$ Similarly, poor sleep has been linked with the upregulation of TNF-a and IL-6 mRNA expression ${ }^{43}$ and promoted the activation of nuclear factor (NF)-kB, which is a transcription factor that plays an important role in promoting the expression of pro-inflammatory genes. ${ }^{20}$ Our findings generalize prior laboratory findings to show that the decline in sleep quality is associated with inflammatory competence, suggesting a possible mechanism relating poor sleep to susceptibility to inflammatory diseases, such as CVDs. ${ }^{44}$ CVDs remain the main cause of death in many developed and developing countries. Research from the past decades has shown that inflammation plays a key role in the progression of CVDs. ${ }^{41}$ The atherosclerotic plaque in the vasculature is known as a complex inflammatory reaction of the vascular wall with inflammatory stimulation from the circulating blood. Therefore, pro-inflammatory cytokines may provide a pathway linking poor sleep to an increased risk of inflammatory diseases, such as CVDs. In summar, previous studies and our findings may explain this association with the consistent results of the activation of immune cells and the elevation of serum inflammatory levels in populations with poor sleep.

In addition, some of our findings diverge from those of previous studies, which showed that elevated CRP levels tended to be more consistently associated with worse sleep quality. ${ }^{45-48}$ These inconsistent findings can be at least partly explained by the different study designs used and study populations selected, the hypotheses being investigated, the inherent challenges of obtaining epidemiologic measurements of intricate and the different methods of analyzing data. In our findings, the global sleep score was associated with CRP levels after adjustments were made for age, gender, race, marital status, education, current smoking status, BMI, and physician-diagnosed CVDs and respiratory diseases, but after perceived stress was then added into Model 2, the global sleep score was no longer related to CRP levels. The difference is that in previous studies, adjustments were not made for perceived stress. ${ }^{45-48}$ Perceived stress may reduce the correlation between poor sleep and CRP levels. ${ }^{49}$ Continued research would need to elucidate the reason. In summary, our results showed a significant negative correlation between sleep quality and serum levels of proinflammatory factors in the obese population.

\section{Limitations}

Finally, there are some limitations in our findings. To participate in this study, all participants needed to be healthy enough to go to a MIDUS study research center, thus leading to the potential for bias. Additionally, the PSQI is used for assessing participants' self-reported subjective sleep quality. A more objective tool is necessary to assess the association between sleep quality and inflammatory biomarkers.

\section{Conclusions}

Poor sleep is positively associated with serum pro-inflammatory marker levels among obese adults. Pro-inflammatory cytokines may provide a pathway linking poor sleep to an 
increased risk of inflammatory diseases. Sufficient sleep may be particularly important for obese adults to prevent chronic health problems, such as CVDs.

\section{Acknowledgment}

Thank you for Ying Jiang's help with the data processing and analysis.

\section{Disclosure}

The authors report no conflicts of interest in this work.

\section{References}

1. Hammond CE. SOME PRELIMINARY FINDINGS ON PHYSICAL COMPLAINTS FROM A PROSPECTIVE STUDY OF 1,064,004 MEN AND WOMEN. Am J Public Health Nations Health. 1964;54 (1):11-23. doi:10.2105/AJPH.54.1.11

2. Gangwisch JE, Heymsfield SB, Bodenalbala B, et al. Short sleep duration as a risk factor for hypertension analyses of the first National Health and Nutrition Examination Survey. Hypertension. 2006;47(5):833-839. doi:10.1161/01.HYP.0000217362.34748.e0

3. Patel SR, Malhotra A, White DP, et al. Association between reduced sleep and weight gain in women. Am J Epidemiol. 2006;164 (10):947-954. doi:10.1093/aje/kwj280

4. Patel SR. Social and demographic factors related to sleep duration. Sleep. 2007;30(9):1077-1078. doi:10.1093/sleep/30.9.1077

5. Sun W, Ling J, Zhu X, et al. Associations of weekday-to-weekend sleep differences with academic performance and health-related outcomes in school-age children and youths. Sleep Med Rev. 2019;46:27-53. doi:10.1016/j.smrv.2019.04.003

6. Drapeau CW, Nadorff MR, McCall WV, et al. Screening for suicide risk in adult sleep patients. Sleep Med Rev. 2019;46:17-26. doi:10.10 16/j.smrv.2019.03.009

7. Wolfson AR, Carskadon MA. Early school times affect sleep and daytime functioning in adolescents. Sleep Res. 1996;25:117.

8. Telzer EH, Fuligni AJ, Lieberman MD, et al. The effects of poor quality sleep on brain function and risk taking in adolescence. NeuroImage. 2013;71(Complete):275-283. doi:10.1016/j.neuroimage.2013.01.025

9. Riemann D, Berger M, Voderholzer U. Sleep and depression - results from psychobiological studies: an overview. Biol Psychol. 2001;57 (1-3):67-103. doi:10.1016/S0301-0511(01)00090-4

10. Bonnet MH. Effect of sleep disruption on sleep, performance, and mood. Sleep. 1985;8(1):11. doi:10.1093/sleep/8.1.11

11. Ross R. Atherosclerosis-an inflammatory disease. $N$ Engl $J$ Med. 1999;340(2):115-126. doi:10.1056/NEJM199901143400207

12. Libby P. Inflammation in atherosclerosis. Nature. 2002;420 (6917):868-874. doi:10.1038/nature01323

13. Cappuccio FP, Cooper D, D'Elia L, et al. Sleep duration predicts cardiovascular outcomes: a systematic review and meta-analysis of prospective studies. Eur Heart J. 2011;32(12):1484-1492. doi:10.10 93/eurheartj/ehr007

14. Ayas NT, White DP, Manson JAE, et al. A prospective study of sleep duration and coronary heart disease in women. Arch Intern Med. 2003;163(2):205-209. doi:10.1001/archinte.163.2.205

15. Hall MH, Muldoon MF, Jennings JR, et al. Self-reported sleep duration is associated with the metabolic syndrome in midlife adults. Sleep. 2008;31(5):635-643. doi:10.1093/sleep/31.5.635

16. Lagrand WK, Visser CA, Hermens WT, et al. C-reactive protein as a cardiovascular risk factor: more than an epiphenomenon? Circulation. 1999;100(1):96-102. doi:10.1161/01.CIR.100.1.96
17. Ridker P. Comparison of C-reactive protein and low-density lipoprotein cholesterol levels in the prediction of first cardiovascular events. N Engl J Med. 2002;347(20):1557. doi:10.1056/NEJMoa021993

18. Danesh J. Low grade inflammation and coronary heart disease: prospective study and updated meta-analyses. BMJ. 2000;321 (7255):199-204. doi:10.1136/bmj.321.7255.199

19. Irwin MR. Why sleep is important for health: a psychoneuroimmunology perspective. Аnпи Rev Psychol. 2015;66(1):143-172. doi:10.1146/annurev-psych-010213-115205

20. Irwin MR, Wang M, Ribeiro D, et al. Sleep loss activates cellular inflammatory signaling. Biol Psychiatry. 2008;64(6):538-540. doi:10. 1016/j.biopsych.2008.05.004

21. Dienberg Love G, Seeman TE, Weinstein M, et al. Bioindicators in the MIDUS National Study: protocol, measures, sample, and comparative context. J Aging Health. 2010;22(8):1059-1080. doi:10.11 $77 / 0898264310374355$

22. Gruenewald TL, Karlamangla AS, Hu P, et al. History of socioeconomic disadvantage and allostatic load in later life. Soc Sci Med. 2012;74(1):75-83. doi:10.1016/j.socscimed.2011.09.037

23. Buysse DJ, Iii CFR, Monk TH, et al. The Pittsburgh sleep quality index: a new instrument for psychiatric practice and research. Psychiatry Res. 1989;28(2):193-213. doi:10.1016/0165-1781(89)90 047-4

24. Cohen S. A global measure of perceived stress. J Health Soc Behav. 1983;24. doi:10.2307/2136404

25. Gangwisch JE, Malaspina D, Posner K, et al. Insomnia and sleep duration as mediators of the relationship between depression and hypertension incidence. Am J Hypertens. 2010;23(1):62-69. doi:10. 1038/ajh.2009.202

26. Newman AB, Spiekerman CF, Enright P, et al. Daytime sleepiness predicts mortality and cardiovascular disease in older adults. The Cardiovascular Health Study Research Group. J Am Geriatr Soc. 2000;48(2):115-123. doi:10.1111/j.1532-5415.2000.tb03901.x

27. Liu Y. Overtime work, insufficient sleep, and risk of non-fatal acute myocardial infarction in Japanese men. Occup Environ Med. 2002;59 (7):447-451. doi:10.1136/oem.59.7.447

28. Davey Smith G, Hart C, Heslop P, et al. Sleep duration and mortality: the effect of short or long sleep duration on cardiovascular and allcause mortality in working men and women. Sleep Med. 2002;3 (4):305-314. doi:10.1016/S1389-9457(02)00016-3

29. Meisinger C, Heier M, L?Wel H, et al. Sleep duration and sleep complaints and risk of myocardial infarction in middle-aged men and women from the general population: the MONICA/KORA Augsburg cohort study. Sleep. 2007;30(9):1121-1127. doi:10.1093/ sleep/30.9.1121

30. Shankar A, Koh WP, Yuan JM, et al. Sleep duration and coronary heart disease mortality among Chinese adults in Singapore: a population-based cohort study. Am J Epidemiol. 2008;168(12):1367-1373. doi:10.1093/aje/kwn281

31. Grandner MA, Hale L, Moore M, et al. Mortality associated with short sleep duration: the evidence, the possible mechanisms, and the future. Sleep Med Rev. 2010;14(3):0-203. doi:10.1016/j.smrv.2009. 07.006

32. Gallicchio L, Kalesan B. Sleep duration and mortality: a systematic review and meta-analysis. $J$ Sleep Res. 2009;18(2):148-158. doi:10. 1111/jsr.2009.18.issue-2

33. Cappuccio FP, D’Elia L, Strazzullo P, et al. Sleep duration and allcause mortality: a systematic review and meta-analysis of prospective studies. Sleep. 2010;33(5):585-592. doi:10.1093/sleep/33.5.585

34. Rd RC, Serody L, Okun ML, et al. Protecting sleep, promoting health in later life: a randomized clinical trial. Psychosom Med. 2010;72 (2):178-186. doi:10.1056/NEJM199704033361401

35. Cizza G, Marincola P, Mattingly M, et al. Treatment of obesity with extension of sleep duration: a randomized, prospective, controlled trial. Clin Trials. 2010;7(3):274-285. doi:10.1177/1740774510368 298 
36. Li JJ, Fang CH, Hui RT. Is hypertension an inflammatory disease? Med Hypotheses. 2005;64(2):236-240. doi:10.1016/j.mehy.2004.06. 017

37. Coles B, Fielding CA, Rose-John S, et al. Classic interleukin-6 receptor signaling and interleukin- 6 trans-signaling differentially control angiotensin ii-dependent hypertension, cardiac signal transducer and activator of transcription-3 activation, and vascular hypertrophy in vivo. Am J Pathol. 2007;171(1):0-325. doi:10.2353/ajpath.2007. 061078

38. Hu FB, Meigs JB, Li TY, et al. Inflammatory markers and risk of developing type 2 diabetes in women. Diabetes. 2004;53(3):693-700. doi:10.2337/diabetes.53.3.693

39. Ridker PM, Cushman M, Stampfer MJ, et al. Inflammation, aspirin, and the risk of cardiovascular disease in apparently healthy men. $N$ Engl J Med. 1997;336(14):973-979. doi:10.1056/NEJM199704033 361401

40. Jonk AM, Houben AJHM, Jongh RTD, et al. Microvascular dysfunction in obesity: a potential mechanism in the pathogenesis of obesityassociated insulin resistance and hypertension. Physiology. 2007;22 (4):252. doi:10.1152/physiol.00012.2007

41. Prather AA, Marsland AL, Hall M, et al. Normative variation in selfreported sleep quality and sleep debt is associated with stimulated pro-inflammatory cytokine production. Biol Psychol. 2009;82(1):017. doi:10.1016/j.biopsycho.2009.04.008
42. Uthgenannt D, Schoolmann D, Pietrowsky R, et al. Effects of sleep on the production of cytokines in humans. Psychosom Med. 1995;57 (2):97-104. doi:10.1097/00006842-199503000-00001

43. Irwin MR. Sleep deprivation and activation of morning levels of cellular and genomic markers of inflammation. Arch Intern Med. 2006;166(16):1756. doi:10.1001/archinte.166.16.1756

44. Esquifino AI, Cardinali DP. Sleep and the immune system. Curr Immunol Rev. 2012;8(1).

45. Larkin EK. Variation of C-reactive protein levels in adolescents: association with sleep-disordered breathing and sleep duration. Circulation. 2005;111(15):1978-1984. doi:10.1161/01.CIR.00001618 19.76138.5E

46. Meier-Ewert HK, Ridker PM, Rifai N, et al. Absence of diurnal variation of $\mathrm{C}$-reactive protein concentrations in healthy human subjects. Clin Chem. 2001;47(3):426-430.

47. Glynn RJ, Macfadyen JG, Ridker PM. Tracking of high-sensitivity C-reactive protein after an initially elevated concentration: the JUPITER Study. Clin Chem. 2008;55(2):305-312. doi:10.1373/ clinchem.2008.120642

48. Liao JK. Genetically elevated C-reactive protein and ischemic vascular disease. $N$ Engl J Med. 2009;11(4):245.

49. Lavretsky H, Newhouse PA. Stress, inflammation, and aging. Am J Geriatr Psychiatry. 2012;20(9):729-733. doi:10.1097/JGP.0b013e31 $826573 \mathrm{cf}$

\section{Publish your work in this journal}

Nature and Science of Sleep is an international, peer-reviewed, open access journal covering all aspects of sleep science and sleep medicine, including the neurophysiology and functions of sleep, the genetics of sleep, sleep and society, biological rhythms, dreaming, sleep disorders and therapy, and strategies to optimize healthy sleep.
The manuscript management system is completely online and includes a very quick and fair peer-review system, which is all easy to use. Visit http://www.dovepress.com/testimonials.php to read real quotes from published authors. 